\title{
Rational design of Two-dimensional Binary Polymers from Hetero Triangulenes for Photocatalytic Water Splitting
}

Zhenpei Zhou, ${ }^{\dagger}$ Maximilian A. Springer, ${ }^{\dagger} \S$ Weixiang Geng, ${ }^{\dagger}$ Xinyue Zhu, ${ }^{\dagger}$ Tianchun $\mathrm{Li}^{\dagger}{ }^{\dagger}$ Manman Li, ${ }^{\dagger}$ Yu Jing, ${ }^{* \dagger}$ and Thomas Heine ${ }^{*}, \AA$

†Jiangsu Co-Innovation Centre of Efficient Processing and Utilization of Forest Resources, College of Chemical Engineering, Nanjing Forestry University, Nanjing 210037, China

tTU Dresden, Fakultät Chemie und Lebensmittelchemie, Bergstraße 66c, 01062 Dresden, Germany"

${ }^{\S}$ Helmholtz-Zentrum Dresden-Rossendorf, Institute of Resource Ecology, Permoserstraße 15, 04318 Leipzig, Germany

*email: yujing@njfu.edu.cn; thomas.heine@tu-dresden.de

Geometry and electronic properties of $2 D$ binary polymers

Table S1. Lattice parameter (L), pore size (P), distance of two in-plane neighboring center atoms $(\mathrm{D})$, formation energy $\left(\mathrm{E}_{\mathrm{f}}\right)$ and band gap $\left(\mathrm{E}_{\mathrm{b}}\right)$ for different $2 \mathrm{D}$ binary HTpolymers in comparison with those of their 2D unary counterparts.

\begin{tabular}{cccccc}
\hline $\begin{array}{c}\text { 2D binary } \\
\text { polymers }\end{array}$ & $\mathrm{L} / \AA$ & $\mathrm{P} / \AA$ & $\mathrm{D} / \AA$ & $\mathrm{E}_{\mathrm{f}} / \mathrm{eV}$ & $\mathrm{E}_{\mathrm{b}} / \mathrm{eV}$ \\
\hline CTPAB & 17.47 & 11.86 & 10.09 & 1.56 & 2.12
\end{tabular}




\begin{tabular}{llllll} 
ОТРАВ & 17.15 & 12.92 & 9.90 & 1.59 & 1.98 \\
МТРАВ & 17.53 & 13.01 & 10.12 & 1.38 & 2.28 \\
CTPA & 17.36 & 11.75 & 10.02 & 1.74 & 2.46 \\
СТРВ & 17.58 & 11.98 & 10.15 & 1.63 & 2.20 \\
ОТРА & 17.07 & 12.71 & 9.86 & 1.49 & 1.80 \\
ОТРВ & 17.17 & 12.80 & 9.91 & 1.86 & 2.83 \\
МТРА & 17.42 & 12.90 & 10.06 & 1.36 & 2.40 \\
МТРВ & 17.62 & 13.10 & 10.17 & 1.54 & 2.84 \\
\hline
\end{tabular}
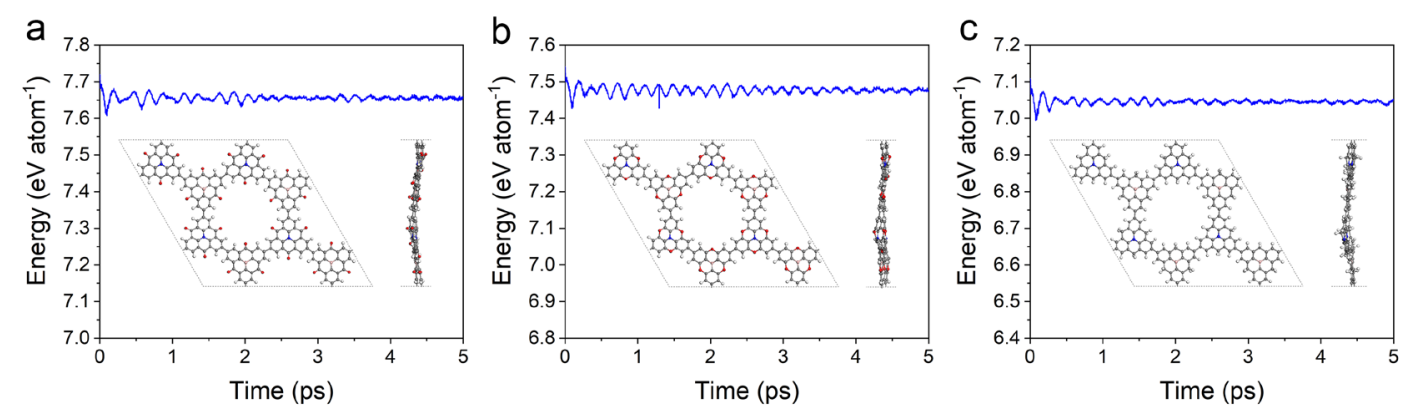

Figure S1. The change of total energy with time of (a) CTPAB (b) OTPAB and (c) MTPAB monolayer by Born-Oppenheimer molecular dynamic (BOMD) simulations at $500 \mathrm{~K}$. Inside are top and side views of an BOMD snapshot of 2D binary HTpolymers at $\mathrm{T}=500 \mathrm{~K}$ after $5 \mathrm{ps}$.

In order to study the band gap opening in the heterotrianguelene polymers, a simple honeycomb tight-binding model is considered:

$$
H=\sum_{i \alpha} \varepsilon_{i} c_{i \alpha}^{\dagger} c_{i \alpha}+\sum_{\langle i, j\rangle \alpha} t_{1} c_{i \alpha}^{\dagger} c_{j \alpha}+\sum_{\langle\langle i, j\rangle\rangle \alpha} t_{2} c_{i \alpha}^{\dagger} c_{j \alpha}
$$

with the on-site energy $\varepsilon$, the first-neighbor hopping energy $t_{1}$, and the secondneighbor energy $t_{2}$. In both cases for equal and non-equal on-site enegies, a firstneighbor hopping of $t_{1}=-0.02$ and a second-neighbor hopping of $t_{2}=-0.0005$ 
are used. In order to model the case with center atoms of different species, the on-site energies of boron and nitrogen atoms are taken from the matci parameters, ${ }^{1}$ being $\varepsilon_{\mathrm{B}}=-0.131903 \mathrm{H}$ and $\varepsilon_{\mathrm{N}}=-0.261222 \mathrm{H}$.

\section{Carrier mobilities and absorption spectrum of $2 D$ binary polymers}

The intrinsic carrier mobility was described based on the deformation potential (DP) theory at the GGA-PBE level of theory according to: ${ }^{2,3}$

$$
\mu_{2 D}=\frac{2 e \hbar^{3} C_{2 \mathrm{D}}}{3 k_{\mathrm{B}} T\left|m^{*}\right|^{2} E_{1}^{2}}
$$

where $m^{*}, C_{2 \mathrm{D}}$ and $E_{1}$ are the effective mass, elastic modulus and deformation potential, respectively. According to the deformation theory, $m^{*}$ and $C_{2 \mathrm{D}}$ describe the band curve and in-plane rigidity for $2 \mathrm{D}$ system. On the other hand, $E_{1}$ is the first derivative of energy versus strain, denoting the shift of band edges. Besides, $\hbar$ is the reduced Planck constant, $k_{\mathrm{B}}$ is Boltzmann constant, and $T$ is the temperature (set to be $300 \mathrm{~K}$ ).

According to $m^{*}=\hbar\left[\partial^{2} E(k) / \partial k^{2}\right]^{-1}$, effective mass for electrons and holes along the armchair and zigzag directions were calculated. The results were shown in Table 1 and Table S2. All the three structures show lighter electrons and holes along the armchair direction compared with those along the zigzag direction. According to $C_{2 \mathrm{D}}=$ $\left[\partial^{2} \mathrm{E} / \partial \delta^{2}\right] / \mathrm{S}_{0}$, the in-plane stiffness $C_{2 \mathrm{D}}$ was calculated by fitting the parabolic relationship between the total energy change and uniaxial strain of the $2 \mathrm{D}$ structures, where $\mathrm{S}_{0}$ is the surface area of the optimized supercell. The applied strain was indicated by Figure S1b. It shows that the energy differences induced by strain are almost identical along the armchair and zigzag directions, resulting in $C_{2 \mathrm{D}}$ values of 58.87 and $59.60 \mathrm{~N} \mathrm{~m}^{-1}$ for 2D CTPAB along the zigzag and armchair directions respectively. 2D OTPAB and MTPAB show comparable values of $C_{2 \mathrm{D}}$, which were illustrated in Table $\mathrm{S} 2$. These results also indicate that the designed $2 \mathrm{D}$ binary polymers possess good inplane stability. The DP constant $E_{1}$ was then calculated by $\left(\partial \mathrm{E}_{\text {edge }} / \partial \delta\right)$ where $\mathrm{E}_{\text {edge }}$ is the band edge of VBM and CBM and $\delta$ represents the strain exertion. As presented in Table 
S1, 2D CTPAB, OTPAB and MTPAB possess small DP constants. Especially, the $E_{1}$ value for $2 \mathrm{D}$ CTPAB is as small as 0.25 and $0.21 \mathrm{eV}$ for electrons along the zigzag and armchair directions, which agrees with the cases of 2D CTPA and CTPB as studied previously. ${ }^{4}$ Finally, the carrier mobility was calculated based on the obtained $\left|m^{*}\right|, C_{2 \mathrm{D}}$, $\left|E_{1}\right|$ and the results are shown in Table S2.

a

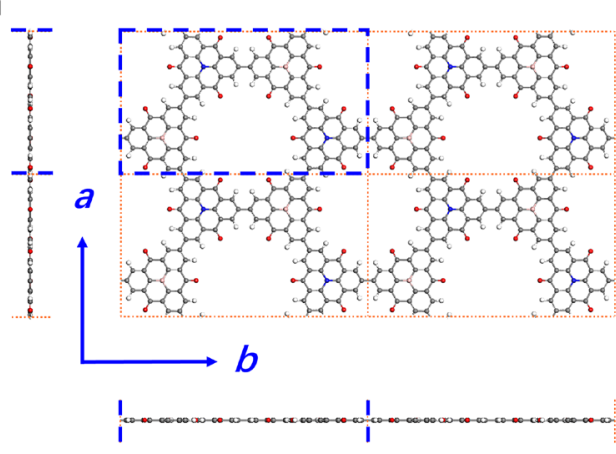

C

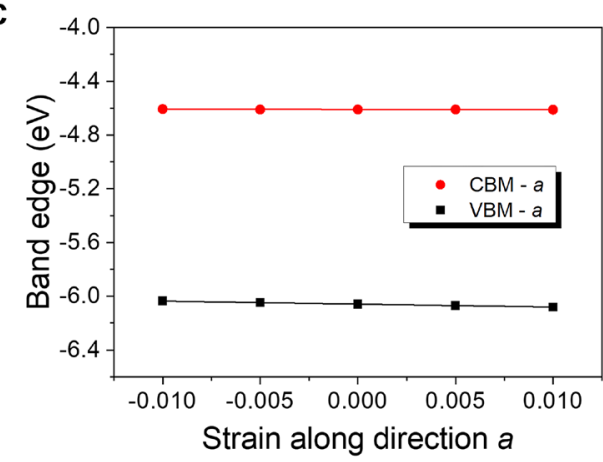

b

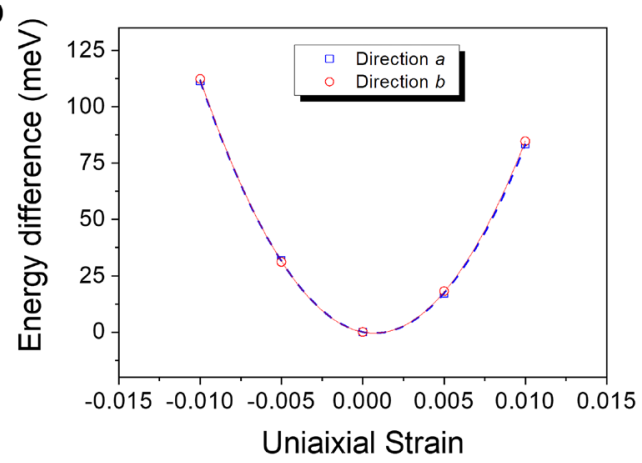

d

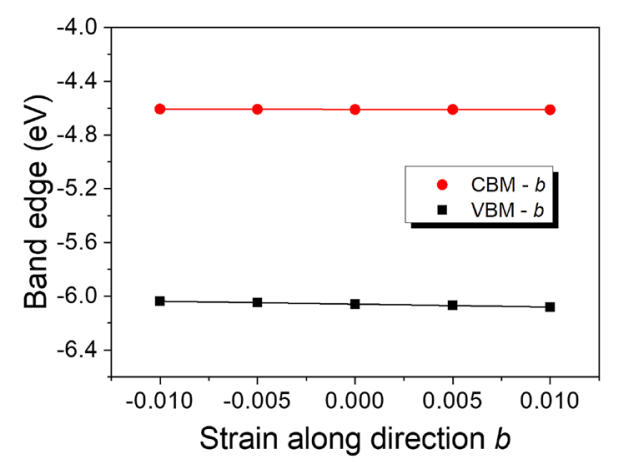

Figure S2. Simulations of the carrier mobility of 2D CTPAB in (a) a rectangular lattice along $a$ (zigzag) and $b$ (armchair) directions; (b) Parabolic fitting along zigzag and armchair directions for $C_{2 \mathrm{D}}$; (c) (d) Energy shift of the VBM and CBM with the lattice stretching and compressing along zigzag and armchair directions.

Table S2. In-plane stiffness $\left(C_{2} \mathrm{D}\right)$, DP constant $\left(\left|E_{1}\right|\right)$, effective mass $\left(\left|m^{*}\right|\right)$ of electrons and holes, and carrier mobilities $(\mu)$ for 2D binary polymers along the zigzag direction. The carrier mobilities were calculated at the PBE level of theory and $m_{0}$ indicates the mass of a single electron.

$$
\text { 2D binary } \quad C_{2 \mathrm{D}} / \mathrm{N} \mathrm{m}^{-1} \quad\left|E_{1}\right| / \mathrm{eV} \quad\left|m^{*}\right| / m_{0} \quad \mu / \times 10^{3} \mathrm{~cm}^{2}
$$




\begin{tabular}{|c|c|c|c|c|}
\hline polymers & & & & $\mathrm{V}^{-1} \mathrm{~s}^{-1}$ \\
\hline \multirow{3}{*}{ СТРАВ } & & 0.25 & $3.30(e)$ & 1.26 \\
\hline & 58.87 & & & \\
\hline & & 2.18 & $1.68(h)$ & 0.063 \\
\hline \multirow{3}{*}{ ОТРАВ } & & 2.58 & $1.20(e)$ & 0.096 \\
\hline & 65.06 & & & \\
\hline & & 3.60 & $1.72(h)$ & 0.024 \\
\hline \multirow{3}{*}{ МТРАВ } & & 2.39 & $1.38(e)$ & 0.081 \\
\hline & 61.64 & & & \\
\hline & & 3.25 & $1.62(h)$ & 0.032 \\
\hline
\end{tabular}

The absorption spectrum of 2D binary polymers was computed at the HSE06 level of theory to describe the ability to capture visible light. The imaginary part $\varepsilon_{2}(\omega)$ of the dielectric function was calculated on the basis of equation: ${ }^{5}$

$$
\varepsilon_{\alpha \beta}^{2}(\omega)=\frac{4 \pi^{2} e^{2}}{\Omega} \lim _{q \rightarrow 0} \frac{1}{q^{2}} \sum_{c, v, \vec{k}} 2 w_{\vec{k}} \delta\left(\varepsilon_{c \vec{k}}-\varepsilon_{v \vec{k}}-\omega\right) \times\left\langle\mu_{c \vec{k}+e_{a \vec{q}}} \mid \mu_{v \vec{k}}\right\rangle\left\langle\mu_{c \vec{k}+e_{\beta \vec{q}}} \mid \mu_{v \vec{k}}\right\rangle^{*}
$$

$c$ and $v$ indicate the conduction and valence band states respectively, and $\mu_{c \vec{k}}$ is the cell periodic part of the orbitals at the $k$-point $\vec{k}$.

\section{Solar driven water splitting processes on $2 D$ binary polymers}

The thermodynamics of water splitting on 2D binary polymers were estimated by examining the HER and OER processes, respectively. We marked *, $\mathrm{H}^{*}, \mathrm{OH}^{*}, \mathrm{O}^{*}$, $\mathrm{OOH}^{*}$ as the bare surface and HER, OER intermediates adsorbed on the surface of 2D binary polymers.

For HER, the two-electron process can be described as two elementary steps:

$$
\text { Step 1: } *+\mathrm{H}^{+}+\mathrm{e}^{-} \rightarrow \mathrm{H}^{*}
$$




$$
\text { Step 2: } \mathrm{H}^{*}+\mathrm{H}^{+}+\mathrm{e}^{-} \rightarrow *+\mathrm{H}_{2}
$$

While for OER, the four-electron process is interpreted as four elementary steps:

$$
\begin{aligned}
& \text { Step 1: }{ }^{*}+\mathrm{H}_{2} \mathrm{O} \rightarrow \mathrm{OH}^{*}+\mathrm{H}^{+}+\mathrm{e}^{-} \\
& \text {Step 2: } \mathrm{OH}^{*} \rightarrow \mathrm{O}^{*}+\mathrm{H}^{+}+\mathrm{e}^{-} \\
& \text {Step 3: } \mathrm{O}^{*}+\mathrm{H}_{2} \mathrm{O} \rightarrow \mathrm{OOH}^{*}+\mathrm{H}^{+}+\mathrm{e}^{-} \\
& \text {Step 4: } \mathrm{OOH}^{*} \rightarrow *+\mathrm{O}_{2}+\mathrm{H}^{+}+\mathrm{e}^{-}
\end{aligned}
$$

By employing the computational hydrogen electrode (CHE) model developed by Nørskov et al, ${ }^{6}$ the Gibbs free energy change $(\Delta G)$ was computed for each elementary step. $\Delta G$ is defined by:

$$
\Delta G=\Delta E+\Delta E_{\mathrm{zpe}}-T \Delta S+\Delta G_{\mathrm{U}}+\Delta G_{\mathrm{pH}}
$$

$\Delta E, \Delta E_{\text {zpe }}$ and $\Delta S$ is the total energy difference obtained from DFT calculations, zeropoint energy change and energy change in entropy at $298.15 \mathrm{~K} . \Delta G_{\mathrm{U}}$ refers to the Gibbs free energy change imposed by light-induced driven potential $(U)$ and equals to -e $U$. The light-induced driven potential for HER can be obtained by $U_{\mathrm{e}}=\left(E_{\mathrm{CBM}}+4.44\right) / \mathrm{e}$, and the driven potential for OER is determined by $U_{\mathrm{h}}=-\left(E_{\mathrm{VBM}}+4.44\right) / \mathrm{e} . E_{\mathrm{VBM}}$ and $E_{\mathrm{CBM}}$ represents the energy level of valance band maximum (VBM) and conduction band minimum $(\mathrm{CBM})$ of $2 \mathrm{D}$ binary polymers, respectively. Both of the VBM and $\mathrm{CBM}$ were regulated by the vacuum level at $\mathrm{pH}=0$. As a result, the free energy change of elementary steps of HER and OER can be described as:

$$
\begin{aligned}
& \Delta G_{1}=G\left(\mathrm{H}^{*}\right)-G(*)-1 / 2 G\left(\mathrm{H}_{2}\right)-\Delta G_{\mathrm{U}}+\Delta \mathrm{G}_{\mathrm{pH}} \\
& \Delta G_{2}=G(*)-G\left(\mathrm{H}^{*}\right)+1 / 2 G\left(\mathrm{H}_{2}\right)-\Delta G_{\mathrm{U}}+\Delta G_{\mathrm{pH}} \\
& \Delta G_{3}=1 / 2 G\left(\mathrm{H}_{2}\right)+G\left(\mathrm{OH}^{*}\right)-G(*)-G\left(\mathrm{H}_{2} \mathrm{O}\right)-\Delta G_{\mathrm{U}}-\Delta \mathrm{G}_{\mathrm{pH}} \\
& \Delta G_{4}=1 / 2 \mathrm{G}\left(\mathrm{H}_{2}\right)+\mathrm{G}\left(\mathrm{O}^{*}\right)-\mathrm{G}\left(\mathrm{OH}^{*}\right)-\Delta \mathrm{G}_{\mathrm{U}}-\Delta \mathrm{G}_{\mathrm{pH}} \\
& \Delta G_{5}=1 / 2 G\left(\mathrm{H}_{2}\right)+G\left(\mathrm{OOH}^{*}\right)-G\left(\mathrm{O}^{*}\right)-G\left(\mathrm{H}_{2} \mathrm{O}\right)-\Delta G_{\mathrm{U}}-\Delta G_{\mathrm{pH}} \\
& \Delta G_{6}=1 / 2 G\left(\mathrm{H}_{2}\right)+G(*)+G\left(\mathrm{O}_{2}\right)-G\left(\mathrm{OOH}^{*}\right)-\Delta G_{\mathrm{U}}-\Delta G_{\mathrm{pH}}
\end{aligned}
$$


The potential of $\mathrm{H}^{+}+\mathrm{e}^{-} \rightarrow 1 / 2 \mathrm{H}_{2}$ under standard conditions $(\mathrm{pH}=0)$ was set to be equilibrium with $0 \mathrm{~V}$ relative to the standard hydrogen electrode (SHE). For those reactions involving the release of protons and electrons, the free energy of one pair of proton and electron $\left(\mathrm{H}^{+}+\mathrm{e}^{-}\right)$under standard conditions was taken as $1 / 2 G\left(\mathrm{H}_{2}\right)$. The entropies of the free molecules, such as $\mathrm{H}_{2}, \mathrm{H}_{2} \mathrm{O}$, were referenced to the NIST database. $^{7}$ As the DFT method cannot accurately describe the high-spin ground state of the $\mathrm{O}_{2}$ molecule, the Gibbs free energy of $\mathrm{O}_{2}\left[G\left(\mathrm{O}_{2}\right)\right]$ is obtained by $G_{\mathrm{O}_{2}}=2 G_{\mathrm{H}_{2} \mathrm{O}}-2 G_{\mathrm{H}_{2}}+4.92$.

The thermodynamic activity of HER and OER can be visualized by examining the overpotential of the reaction $(\eta)$, which is determined by:

$$
\begin{aligned}
& \eta_{\mathrm{HER}}=\max \left\{\Delta G_{1}, \Delta G_{2}\right\} / \mathrm{e} \\
& \eta_{\mathrm{OER}}=\max \left\{\Delta G_{3}, \Delta G_{4}, \Delta G_{5}, \Delta G_{6}\right\} / \mathrm{e}-1.23
\end{aligned}
$$

$\Delta G_{1} \sim \Delta G_{6}$ represent the free energy change of the elementary steps in equation (11) (16), respectively. A smaller $\eta$ indicates a higher activity of the catalyst.

In this work, the $\mathrm{pH}$ of the solution is assumed to be zero for an acid medium and the free energy contribution caused by changing the $H$ concentration is neglected $\left(\Delta G_{\mathrm{pH}}\right.$ $=0$ ). As a result, by shifting the energy of these charge involved steps by $\Delta G_{\mathrm{U}}=-\mathrm{e} U$, the thermodynamics of hydrogen and oxygen evolution will be more favorable. 
a
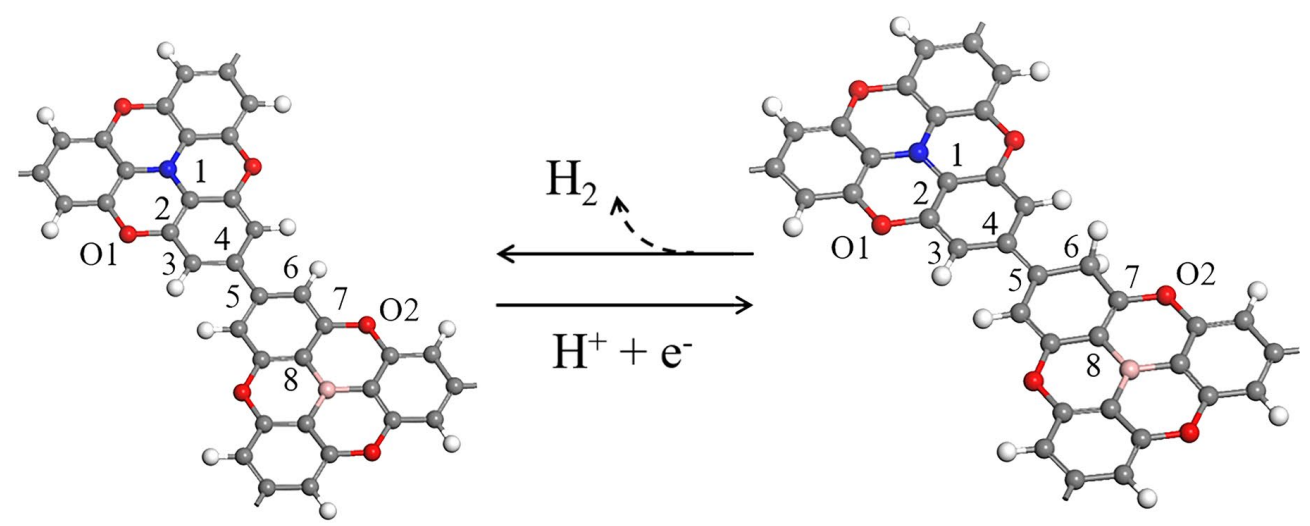

b
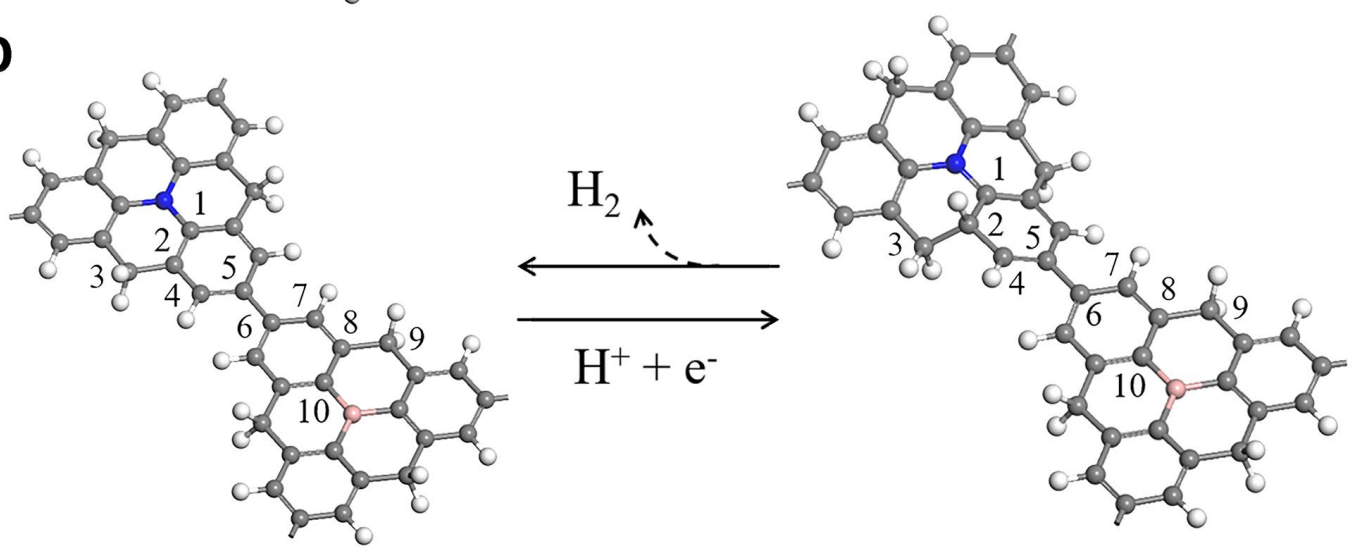

Figure S2. Schematics of HER on OTPAB (a) and MTPAB (b). The reactive site is found to be the $\mathrm{C} 6$ site of OTPAB and $\mathrm{C} 2$ site of MTPAB, respectively.
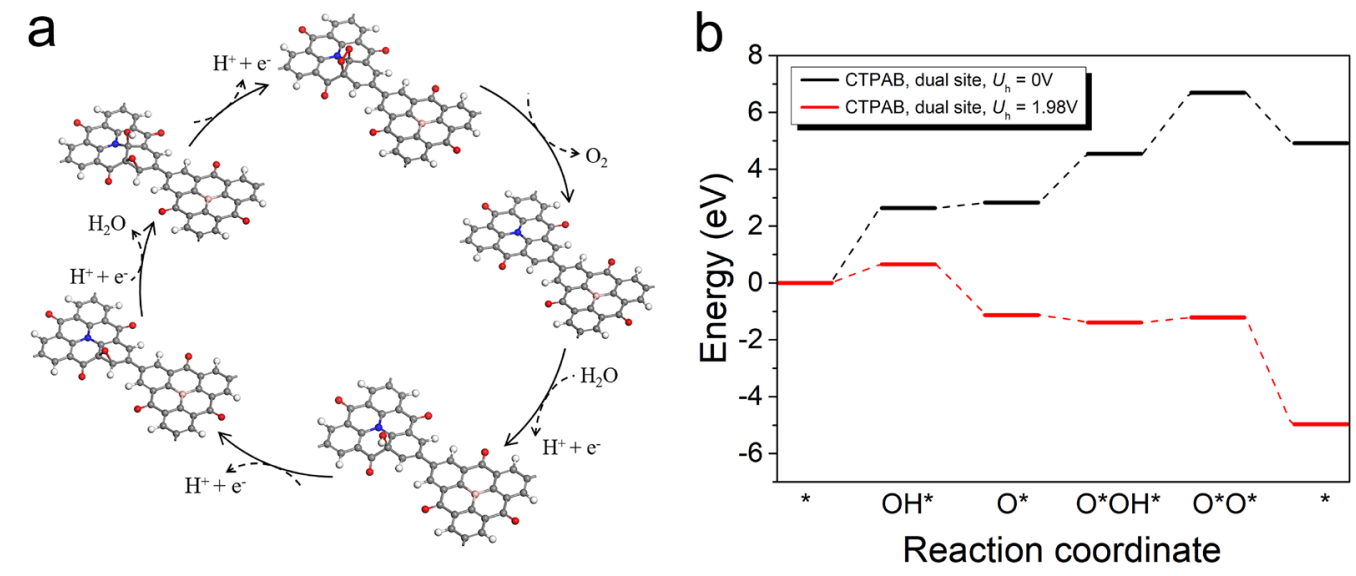

Figure S3. (1) Schematics and (b) free energy profiles of OER following a dual-site process on $\mathrm{CTPAB}$ in the absence of light irradiation $(\mathrm{U}=0 \mathrm{~V})$ and under light irradiation $(\mathrm{U}=1.98 \mathrm{~V})$. 


\section{REFERENCES}

(1) Frenzel, J.; Oliveira, A. F.; Jardillier, N.; Heine, T.; Seifert, G. Semi-relativistic, selfconsistent charge Slater-Koster tables for density-functional based tight-binding (DFTB) for materials science simulations. TU-Dresden 2004-2009.

(2) Cai, Y.; Zhang, G.; Zhang, Y.-W. Polarity-Reversed Robust Carrier Mobility in Monolayer $\mathrm{MoS}_{2}$ Nanoribbons. J. Am. Chem. Soc. 2014, 136, 6269-6275.

(3) Qiao, J.; Kong, X.; Hu, Z. X.; Yang, F.; Ji, W. High-mobility transport anisotropy and linear dichroism in few-layer black phosphorus. Nat. Commun. 2014, 5, 4475.

(4) Jing, Y.; Heine, T. Two-dimensional Kagome Lattices Made of Hetero Triangulenes are Dirac Semimetals or Single-Band Semiconductors. J. Am. Chem. Soc. 2019, 141, 743-747.

(5) Gajdoš, M.; Hummer, K.; Kresse, G.; Furthmüller, J.; Bechstedt, F. Linear optical properties in the projector-augmented wave methodology. Phys. Rev. B 2006, 73, 045112.

(6) Skúlason, E.; Karlberg, G. S.; Rossmeisl, J.; Bligaard, T.; Greeley, J.; Jónsson, H.; Nørskov, J. $K$. Density functional theory calculations for the hydrogen evolution reaction in an electrochemical double layer on the Pt(111) electrode. Phys. Chem. Chem. Phys. 2007, 9, 3241-3250.

(7) NIST Computational Chemistry Comparison and Benchmark Database, NIST Standard Reference Database Number 101, Release 21, August 2020, edited by Russell D. Johnson, III, Available at http://cccbdb.nist.gov/. 